Casillas, Barbero y Moreno (2013) / Revista de Empresa Familiar, 3(1), 7-20.

\title{
Reestructuración y tipo de propiedad en empresas en crisis. Diferencias entre empresas familiar y no familiares
}

\section{Turnaround Strategy and ownsership structure in declining firms: diferences between family and non-family businesses}

\author{
Jose Carlos Casillas $^{\mathrm{a}^{*}} \cdot$ José Luis Barbero Navarro $^{\mathrm{b}} \cdot$ Ana María Moreno Menéndez $^{\mathrm{a}}$ \\ ${ }^{a}$ Departamento de Administración de Empresas y Comercialización e Investigación de Mercados (Marketing), \\ Universidad de Sevilla, Sevilla (Spain) \\ ${ }^{b}$ Departamento de Organización de Empresas y Marketing. Facultad de Ciencias Empresariales, Universidad \\ Pablo de Olavide, Sevilla (Spain)
}

\section{A T OS ARTÍCULO}

Historial:

Recibido 09-02-2013

Aceptado 04-05-2013

Palabras clave:

Reestructuración

Declive

Estructura de propiedad,

Empresa familiar

Crisis

Códigos JEL:

L1, L2, M1, M2

A R T I C LE IN F O

Article history:

Received 09-02-2013

Accepted 04-05-2013

Keywords:

Turnaround

Decline

Ownership structure

Family Business

Crisis

JEL codes:

L1, L2, M1, M2

\section{RE S U M EN}

El presente trabajo analiza la influencia de la estructura de propiedad en las estrategias de reestructuración en empresas en crisis, específicamente, en el caso de las pequeñas y medianas empresas. En concreto, nos centramos en el estudio de las diferencias entre empresas familiares y no familiares a la hora de afrontar y reaccionar ante resultados decrecientes. Sobre una muestra de 1.807 empresas españolas (PYMEs), de las que 947 están controladas por una persona o familia, hemos encontrado que las empresas familiares con resultados negativos tienden a mantener o aumentar puestos de trabajo al tiempo que reducen sus activos a través de procesos de desinversión. Asimismo, hemos comprobado que esta relación se da con mayor intensidad en el caso de las empresas familiares de menor tamaño y edad. Finalmente, el nivel de concentración de la propiedad también parece ejercer un papel moderador.

\section{A B S T R A C T}

The present paper analyzes the influence of the ownership structure on turnaround strategies in declining firms, especially in the case of small and medium-sized businesses. Concretely, we focus on the study of the differences between family and non-family businesses when they react to decreasing financial results during few years. Using a sample of 1,807 Spanish firms (SMEs), 947 of them controlled by a single person or a family, we have found that family-owned businesses with negative results tend to maintain or increase the number of employees while they reduce their assets, by divestments operations. We have also confirmed that this relationship is more intense in the case of smaller and younger family-owned businesses. Finally, we have considered concentration of ownership structure as an additional moderator variable in the study. 


\section{Introducción}

La actual crisis mundial ha incrementado notablemente el numero de procesos de concurso de acreedores y cierres de empresas en todo el mundo. Mas de treinta millones de empleos se han perdido en estos ultimo años por la crisis (IMF, 2010). Investigaciones precedentes han puesto de manifiesto que, en cualquier momento, entre un veinte y un treinta por ciento de empresas necesitan de algún tipo de reestructuración (Boyle y Desai, 1991; Murphy, 1986) y que entre un sesenta y un ochenta por ciento de nuevas empresas fracasan (Knott y Posen, 2005). Kierulff y Petersen (2009) confirmaron estos datos recientemente, señalando que las nuevas empresas tienen una tasa de fracaso superior al 65 por ciento en sus primeras etapas de vida.

Examinando estos datos, parece que el fracaso supone un fenómeno importante dentro de la administración de empresas, especialmente en entornos hostiles o ante una crisis económica y financiera como la actual. El fracaso empresarial suele venir precedido de una fase de declive, en el que las empresas pierden ventaja competitiva como consecuencia de factores tanto externos (recesión económica, obsolescencia tecnológica, ineficiencia de factores productivos, etc.) como internos (falta de competencias de gestión, conflictos internos, rigideces organizativas, etc.) (Pearce II y Robbins, 2008). Los procesos de cambio de rumbo suponen el establecimiento de un proceso explicito o implícito en el que se realiza un diagnostico de la situación, se genera información analítica, se desarrollan planes de acción y se evalúan los resultados, con el fin de transformar procesos de declive en periodos de crecimiento o rentabilidad (Di Primio, 1988). A lo largo de la década de los noventa, Pearce II y Robbins (1993; 1994a; 1994b) desarrollaron una conceptualización de estos procesos a través de dos etapas, que ha venido siendo aceptada desde entonces en múltiples investigaciones: (1) fase de ajuste y (2) fase de recuperación.

El presente trabajo analiza la influencia de la estructura de propiedad en las estrategias de ajuste en empresas en crisis, específicamente, en el caso de las pequeñas y medianas empresas.
En concreto, nos centramos en el estudio de las diferencias entre empresas familiares $y$ no familiares a la hora de afrontar y reaccionar ante resultados decrecientes. En este sentido, tanto la teoría de la agencia (Jensen, 1986; 1993) como la dirección estratégica (Rahul, 1996; Rahul y Hitt, 1998) reconocen que la estructura de propiedad y el gobierno corporativo pueden ejercer un impacto relevante en las decisiones estratégicas de la empresa. Sin embargo, apenas se han desarrollado investigaciones de este tipo de influencias en empresas en crisis o con dificultades financieras (Morrow, Johnson y Busenitz, 2004). Cater y Schwab (2008) subrayan la especificidad de la empresa familiar a la hora de enfrentarse a resultados decrecientes, especialmente derivados de los que Gomez-Mejia, Haynes y Nuñez (2007) han venido a denominar el patrimonio socioeconómico de este tipo de empresas.

En la sección siguiente pasamos a exponer el marco teórico, basado en la literatura de reestructuración y de estrategias de cambio de rumbo. El tercer apartado introduce la estructura de propiedad y la especificidad de la empresa familiar, proponiendo un conjunto de hipótesis. Posteriormente, se describe la metodología de la investigación empírica. La quinta sección expone los principales resultados y son analizados a la luz de los enfoque teóricos empleados. Finalmente, en el ultimo epígrafe, se resumen las principales conclusiones, limitaciones y potenciales líneas futuras de investigación.

\section{Marco teórico}

\subsection{Antecedentes históricos}

Una característica de la literatura clásica sobre reestructuraciones en la ausencia de un marco teórico claro. A lo largo de los ultimo quince años, diferentes trabajos han realizado un considerable esfuerzo en sustentar este fenómeno sobre bases teóricas solidas (Mellahi y Wilkinson, 2004; Abor, Graham y Yawson, 2011). En concreto, tres perspectivas han abordado el fenómeno de las estrategias de cambio de rumbo y reestructuraciones: la teoría 
de la agencia, la teoría de recursos y capacidades y la perspectiva de la diversificación (Mellahi y Wilkinson, 2004).

La teoría de la agencia se basa en la idea básica de que la relación entre principal y agente afecta y reflejan como las empresas organizan sus recursos y el grado de Asunción de riesgos de la empresa (Alchian y Demsetz, 1972; Jensen y Meckling, 1976). Este enfoque se centra en el contrato entre el principal y el agente como unidad de análisis esencial, e incluye determinadas asunciones sobre el individuo y sobre la organización a la hora de plantear sus hipótesis. Entre las asunciones individuales se encuentran al interés propio, la racionalidad limitada y la aversión al riesgo. Como asunciones organizativas están el empleo del conflicto, los criterios de efectividad y eficiencia y la asimetría de la información entre agente y principal (Eisenhardt, 1989). Este enfoque ha sido empleado en la investigación sobre reestructuraciones especialmente en el área de los recursos humanos, relacionado con el proceso de sucesión en los equipos directivos (Haynes, Thomson y Wright, 2003). Asimismo, ha sido utilizado en el ámbito financiero, analizo como influye en el ámbito de la estructura de deuda de las empresas.

Penrose (1959) inicialmente propuso que la empresa podía entenderse como un conjunto único de recursos, enfatizó el papel que su combinación a la hora de conformar las características idiosincrásicas de cada organización (Barney, 1991; Harrison, Hitt, Ireland y Hoskisson, 2001). Hofer y Schendel (1978) diferenciaron seis tipos de recursos: financieros, tecnológicos, físicos, humanos, organizativos y de reputación. Otros autores, como Collis y Montgomery (1998), diferenciaron entre tres categorías: recursos tangibles, recursos intangibles y capacidades organizativas. Desde esta perspectiva, diferentes trabajos han analizado la estrategias de reestructuraciones específicamente en el ámbito de los recursos humanos, así como en aspectos ligados al reclutamiento $\mathrm{y}$ promoción de directivos medios de la organización (Mellahi y Wilkinson, 2004).
Una tercera perspectiva que ha analizado el fenómeno de las reestructuraciones el de la diversificación (Rummelt, 1974). Ansoff (1957) desarrolló su conocida matriz producto-mercado como herramienta de análisis de las diferentes alternativas estratégicas de crecimiento (Kotler, 2000). De acuerdo con este enfoque, es posible explicar alternativas mas o menos concentradas o diversificadas así como estrategias de reenfoque o desinversiones orientadas a la concentración es los negocios principales de la organización.

\subsection{Estrategias de reestructuración}

La investigación sobre las estrategias de cambio de rumbo y reestructuración han evolucionado a lo largo del tiempo (Mellahi y Wilkinson, 2004). Los primeros trabajos en este campo se centraron en la búsqueda de recetas o directrices para recuperar niveles de crecimiento aceptables en sectores en declive o maduros. En este sentido, siguiendo los trabajos de Porter (1980), Hall (1980) encontró ciertas evidencias que sugerían que aquellas empresas que se centraban en las estrategias de reducción de costes o de una significativa diferenciación se recuperaban mejor de periodos de declive, especialmente en empresas del sector secundario.

A lo largo de la década de los 90, la literatura empezó a analizar el proceso de reestructuración en si mismo, y se empezó a diferenciar dos etapas o fases: el ajuste y la recuperación (Robbins y Pearce II, 1993). La fase de ajuste es la primera de ellas y consiste en un proceso de reducción drástica de los costes con el fin de evitar la pérdida constante de liquidez, de forma que se alcance una cierta estabilidad financiera. El objetivo básico es dejar de perder dinero. El modo de alcanzar esta estabilidad se centra en la liquidación de activos, las desinversiones, la salida de aquellos negocios no rentables, etc. (Robbins y Pearce II, 1993). Estos autores muestran que la supervivencia de las pequeñas empresas va inexorablemente unida a los resultados de su negocio principal (Robbins y Pearce II, 1992; Pearce II y Robbins, 1994a). Cuando el rendimiento económico de una 
pequeña empresa, con una reducida cuota de mercado, decrece, la estrategia de reestructuración no es, sin embargo, algo tan simple como cambiar de segmento de negocio, siendo necesarias medidas de ajuste en el propio negocio central de la empresa. Pearce II y Robbins (1994b) argumentan que las estrategias de ajuste están relacionadas con el éxito del proceso de reestructuración. Así, el grado en que se produzca una reducción de activos, se relaciona positivamente con el éxito de la recuperación.

Así pues, una conclusión a la que llegan estas investigaciones es que, en el caso de pequeñas empresas productivas, el camino mas eficiente para el desarrollo de una recuperación es atravesar una fase de reducción de costes y activos lo mas decididamente posible. Otros investigadores, como Behn (1980) y D'Aveni (1989) han defendido, no obstante, que se conoce poco sobre cuándo, cómo y de qué modo debería ser utilizado este proceso de ajuste como medio para recuperar los resultados financieros de la empresa. En el contexto de la empresa familiar, Cater y Schwab (2008) han propuesto que los procesos de ajuste y reestructuración han de considerar ocho factores específicos de contingencia que moderan la relación entre ajuste y recuperación. Estos factores son los sistemas de gestión informales, la orientación hacia el consenso, el numero de candidatos para la sucesión, la fortaleza de los lazos, la orientación interna, la integración de los empleados no familiares, las motivaciones altruistas y la orientación a los objetivos a largo plazo.

\section{Reestructuración y Empresa Familiar}

Aunque las empresas familiares tienen numerosas características comparables al del resto de empresas (Sharma, 2004), el tipo de relaciones que se desarrollan en este tipo de compañías entre familiares y entre estos y el resto de empleados y directivos genera un tipo especifico de organización (Chua, Chrisman y Steier, 2003). Numerosos estudios empíricos han puesto de manifiesto que las empresas familiares han de afrontar diversos retos $y$ procesos críticos, mostrando elevadas tasas de fracaso (Ibrahim, Soufani y Lam, 2001; Shanker y Astrachan, 1996). Cater III y Schwab (2008) desarrollaron un estudio cualitativo en relación con el proceso de reestructuración de dos empresas familiares en el que identificaron que el hecho de ser empresas familiares proporcionaban factores habilitadores e inhibidores simultáneamente. Mientras que el fuerte compromiso de la familia puede inhibir la realización de cambios en la alta dirección, las interconexiones entre los miembros de la empresa familiar, su orientación altruista y a la consecución de objetivos a largo plazo supervivencia- pueden incentivar medidas drásticas de reestructuración organizativa (Cater y Schwab, 2008).

Diversas investigaciones sugieren que las empresas familiares son mas flexibles que las no familiares como consecuencia de contar con modelos de organización y gestión mas centralizadas e informales (Nordqvist, Habbershon y Melin, 2008). En este sentido, una corriente de trabajos defiende que estas empresas tienen una mayor capacidad para desarrollar patrones de comportamiento emprendedor, ya que su supervivencia depende de su capacidad para entrar en nuevos mercados en los que ofrecer productos y servicios innovadores (Ward, 1987; Zahra, Hayton, y Salvato, 2004). Casillas, Moreno y Barbero (2010) argumentan que las empresas familiares muestran niveles superiores de centralización y velocidad en los procesos de toma de decisiones. Esto es especialmente en las empresas familiares de primera generación, en las que el fundador de la empresa ocupa una posición central en la organización que le proporciona de poder suficiente para conducir el negocio de acuerdo con su propia visión del mismo (Gedajlovic, Lubatkin y Schulze, 2004).

Así las empresas familiares en primera generación disponen de una mayor libertad de acción gracias al poder y legitimidad que detenta el fundador de la empresas (Mitchell, Hatt, Valcea y Towsend, 2009), que coadyuva a conformar organizaciones con estructuras centralizadas en las que las decisiones se adoptan rápidamente permitiendo 
comportamientos emprendedores por parte de las empresas (Chrisman, Chua y Steier, 2003). El propietario-directivo tiende a manifestar un fuerte liderazgo que permite acelerar los procesos de toma de decisiones. En el caso de que éste muestre una elevada orientación innovadora, la propia organización implantara decisiones de innovación mas rápidamente que empresas no familiares. Incluso en empresas familiares en segunda generación o mas allá, la implicación de miembros de la familia en la gestión de la empresa a través de la coincidencia de accionistas familiares $y$ directivos $o$ consejeros puede igualmente conducir a una perdida de mecanismos de control que permite posicionar y reubicar recursos con bastante agilidad y velocidad (Bammers, Voordeckers y Van Gils, 2011).

De acuerdo con los argumentos expuestos, es posible encontrar en la literatura dos argumentos opuestos en relación con los procesos de reestructuración en empresas familiares respecto a las no familiares. Por un lado, las empresas familiares pueden desarrollar acciones reactivas mas intensas y rápidas como resultado de su superior agilidad cuando sus resultados comienzan a verse afectados por un proceso de declive o crisis como el actual. Desde nuestro punto de vista, la orientación emprendedora de la empresa no solo implica crecimiento o resultados superiores (Moreno y Casillas, 2008), sino que también supone una mayor facilidad para implantar estrategias mas o menos drásticas con el fin de modificar el rumbo de la empresa y desarrollar procesos de reestructuración. La capacidad de emprendimiento entendida como la capacidad de descubrir y explotar oportunidades de negocio suele asociarse al fenómeno del crecimiento en periodos expansivos. Sin embargo, en contextos de recesión o declive como el actual, las estrategias de ajuste $\mathrm{y}$ desinversiones puede proporcionar la llave para la supervivencia a largo plazo de la empresa (Mellahi y Wilkinson, 2004). Por otro lado, los propietarios-directivos de las empresas familiares tienden a mostrar un elevado compromiso con la empresa (con sus empleados, recursos, estrategias, etc.), superior al de aquellas empresas no familiares (Miller,
Breton-Miller y Scholnick, 2008). Este mayor compromiso puede implicar una tendencia a evitar decisiones de ajuste drástico ante resultados negativos, tratando de conservar los puestos de trabajo, sus productos y mercados tradicionales y las estrategias implantadas en el pasado. En muchos casos, las posibilidades reales de sustituir al máximo responsable son escasas y realizar un cambio estratégico profundo puede suponer implícitamente la aceptación de un fracaso en las decisiones adoptadas en el pasado. Teniendo en cuenta por tanto, ambos argumentos, proponemos las dos hipótesis siguientes:

Hipótesis 1a: Ante resultados negativos, las empresas familiares tienden a realizar procesos de ajuste mas intensos que las empresas no familiares.

Hipótesis 1b: Ante resultados negativos, las empresas familiares tienden a realizar procesos de ajuste menos intensos que las empresas no familiares.

Tradicionalmente, tanto el tamaño como la edad de la empresa suelen considerarse variables que ejercen un impacto en el comportamiento empresarial, y la influencia de la estructura de propiedad sobre las estrategias de reestructuración no deben ser una excepción. Cuando una empresa desarrolla este tipo de estrategias, asume que su organización esta sobredimensionada, lo que justifica un proceso de reducción de tamaño y de liquidación de activos. En el caso de las empresas más pequeñas, los trabajadores cuentan con menores niveles de especialización lo que hace mas difícil prescindir de personal para reducir costes de operación. Asimismo, suele considerarse que las empresas mas jóvenes se encuentran en un proceso de búsqueda de su dimensión optima en la que se comience a obtener beneficios o estos sean los esperados. Por el contrario, las empresas maduras puede enfrentarse a problemas de sobrecapacidad (Lieberman, 1987). Este problema emerge cuando las empresas tratan de anticiparse a las oportunidades de negocio expandiendo su capacidad con el fin de acaparar la mayor cuota de mercado posible. (Porter, 1980). Sin embargo, las empresas pueden errar en sus 
expectativas $\mathrm{y}$, al mismo tiempo, diversas empresas pueden ampliar su capacidad simultáneamente de forma que, pasado el tiempo, se encuentren sobredimensionadas (Porter y Spence, 1982). De acuerdo con estos argumentos, esperamos que aquellas empresas con una mayor dimensión y edad serán más agresivas a la hora de implantar medidas de ajustes y reestructuración como reacción a resultados decrecientes que aquellas mas jóvenes y pequeñas. Por esta razón, proponemos:

Hipótesis 2: el tamaño y la edad de la empresa moderara la influencia de la estructura de propiedad sobre la estrategia de reestructuración de la empresa ante resultados negativos, de modo que la influencia del carácter familiar de la empresa y la estrategia de reestructuración será mas intensa en empresas de mayor tamaño y edad que en las más pequeñas y jóvenes

Diferentes trabajos han puesto de manifiesto que no todas las empresas familiares son iguales, existiendo una amplia heterogeneidad entre ellas (Sharma, 2003). En este sentido, cuando la concentración de la propiedad es muy elevada, los propietarios acumulan una gran capacidad y poder de decisión, con niveles muy reducidos de mecanismos de control (Bammens et al., 2011; Schulze, Lubatkin, y Dino, 2003). Este suele ser el caso de las empresas en la etapa del fundador, en el que éste ostenta la mayor capacidad y libertad de decisión, como consecuencia de su superior nivel de legitimidad interna (Mitchell, Hatt, Valcea y Towsend, 2009). En este contexto, la estructura suele ser muy plana, en el que la toma de decisiones tiene a tener un marcado acento informal y muy centralizado en la figura del fundador o de su equipo mas próximo (Chrisman, Chua y Steier, 2003). Durante esta etapa, la empresa cuenta con una gran capacidad para desarrollar cualquier senda estratégica y, llegado el caso, dispone de una amplia discrecionalidad para realizar las modificaciones que sean necesarias (Gedajlovic, Lubatkin y Schulze, 2004). Sin embargo, estos argumentos pueden ser extrapolados también al de aquellas empresas no familiares pero con una elevada concentración en su estructura de propiedad. Por el contrario, cuando la estructura de propiedad de la empresa esta muy diversificada, con un amplio numero de accionistas, suelen establecerse mecanismos de gobierno que incrementen los mecanismos de control con el fin de reducir los problemas de agencia (Jensen y Meckling, 1976). En resumen, cabe esperar que una alta concentración de la propiedad facilita la agilidad estratégica de la empresa, necesaria para desarrollar con eficacia los procesos de ajuste y reestructuración ante resultados crecientemente negativos. Por tanto, proponemos una ultima hipótesis:

Hipótesis 3: la concentración de la propiedad de la empresa moderara la influencia de la estructura de propiedad sobre la estrategia de reestructuración de la empresa ante resultados negativos, de modo que la influencia del carácter familiar de la empresa sobre la estrategia de reestructuración será mas intensa en empresas con mayores niveles de concentración de la propiedad.

\section{Metodología}

\subsection{Muestra}

La investigación empírica se ha desarrollado sobre una muestra de empresas que han mostrado unos resultados negativos a lo largo de un periodo de tres años (2006-2008). La fuente utilizada para la extracción de la muestra ha sido la base de datos SABI (Sistema de Análisis de Balances Ibérico) que comprende la gran mayoría de sociedades mercantiles registradas en España.

La selección de la muestra se hizo considerando tres criterios. en primer lugar, controlamos el tamaño de las empresas, incluyendo sólo aquellas empresas que tuviesen entre 10 y 250 empleados en el momento inicial de dicho periodo, esto es, en el año 2006. Excluimos las empresas menores de 10 empleados ya que éstas pueden ser confundidas con emprendedores en los que el concepto organizacional puede no estar del todo desarrollado (Goldeng, 2008). Asimismo, dejamos fuera a las empresas grandes, con 250 trabajadores o más, ya que las características 
organizativas de este tipo de empresas son muy diferentes a las de la mayoría de PYMEs. En segundo lugar, seleccionamos aquellas empresas con valor medio negativo del EBITDA durante el periodo 2006-2008. Finalmente, seleccionamos aquellas empresas en la que la información sobre su estructura de propiedad estaba disponible en la base de datos. Una vez considerados los tres criterios anteriores, la muestra final estuvo compuesta por un total de 1.807 empresas, de las cuales 947 eran empresas familiares — propiedad familiar $(52,4 \%)$ - 133 cuya propiedad estaba bajo el control de diferentes instituciones financieras, entidades de capital riesgo, business angels, fondos de inversión, etc. - propiedad financiera $(7,3 \%)$ , y 727 cuya propiedad estaba controlada por otras corporaciones o grupos empresariales propiedad corporativa $(40,23 \%)$ -

\subsection{Variables}

\subsubsection{Variables dependientes}

Para medir la intensidad del proceso de reestructuración, hemos empleado dos variables diferentes. La primera es la evolución (crecimiento) del número de empleados en el periodo 2008-2009, esto es, los años posteriores a los de la obtención de los malos resultados (2006-2008). La segunda variable ha sido la evolución de sus activos en los mismos años (2008-2009). Con el fin de evitar la posible influencia del sector, derivada de posibles diferencias en su ciclo de vida, su intensidad tecnológica, etc., ambas variables dependientes han sido centradas respecto a la media de su sector a nivel de dos dígitos de la clasificación nacional de actividades económicas. De este modo, ambas medidas de reestructuración relativizadas sectorialmente son comparables en una muestra multase teorías como la empleada. Asimismo, dichas variables han sido estandarizadas previamente a ser analizados los efectos moderadores.

\subsubsection{Variables independientes}

Hemos diferenciado tres tipos de estructuras de propiedad a través de dos variables dummies (propiedad familiar, propiedad financiera, $\mathrm{y}$ propiedad corporativa). Esta información fue directamente extraída de la base de datos original, en función del principal accionista de cada empresa. Así, una empresa se categoriza como de propiedad familiar si su principal accionista o propietario era una persona física o una familia. Una empresa se considera de propiedad corporativa, si su principal accionista era otra empresa o corporación, salvo que dicha empresa fuese un banco $u$ otro tipo de institución financiera, en cuyo caso se calificó como una empresa de propiedad financiera.

\subsubsection{Variables moderadoras}

El tamaño ha sido medido a través de la media del numero de trabajadores a lo largo del periodo 2006-2008. Con el fin de evitar problemas con la distribución de dicha variables, hemos empleado su logaritmo. De forma similar, la edad de la empresa ha sido medida mediante el logaritmo de la antigüedad de la empresa en 2008 (años desde su fundación). En cuanto a la concentración de la estructura de propiedad, hemos empleado dos variables diferentes. La primera es el número de accionistas de la empresa, y la segunda consiste en el porcentaje del total de acciones concentrado en el principal accionista de la empresa.

\subsubsection{Variables de control}

Hemos utilizado cuatro variables dummies para controlar la posible influencie del sector, correspondientes a fabricación, construcción, comercio y servicios, siendo el sector agrícola el empleado como sector de comparación.

\subsection{Método}

Hemos procedido a estimar diversos modelos de regresión (OLS) con el fin de poder testar las diferentes hipótesis planteadas. Así, para cada una de los dos variables dependientes, siguiendo un análisis de regresión jerárquico, hemos estimado tres modelos diferentes: el primero en el que se han incluido sólo las variables de control (modelo base), posteriormente un segundo modelo ha añadido las variables independientes directas, esto es, la relativa al tipo de propiedad, para pasar, en un tercer 
modelo, a incorporar las interacciones o efectos moderadores.

\section{Resultados}

La tabla 1 resume los principales parámetros descriptivos (media y desviación típica) de las variables, así como las correlaciones entre ellas.

En relación con la evolución del número de empleados, hemos encontrado, en primer lugar, una influencia negativa $\mathrm{y}$ significativa del tamaño $(b=-0.108 ; p<0.01)$ y de la edad de la empresa $(b=-0.110 ; p<0.01)$ sobre el crecimiento del numero de trabajadores. Este resultado pone de manifiesto que las empresas más pequeñas y jóvenes tienden a mantener $\mathrm{o}$ aumentar el numero de trabajadores mientras que las más gres y maduras tienden a reducirlo. En relación con la estructura de propiedad, los resultados encuentran que la propiedad familiar ejerce una influencia significativa y positiva

Tabla 1

Estadísticos descriptivos y matriz de correlación.

\begin{tabular}{|c|c|c|c|c|c|c|c|c|c|}
\hline & Media & Desv. Tip. & 1 & 2 & 3 & 4 & 5 & 7 & 8 \\
\hline l. LnTamaño & 0.379 & 0.922 & 1.000 & & & & & & \\
\hline 2. LnAccionistas & 0.563 & 0.674 & $0.069 * *$ & 1.000 & & & & & \\
\hline 3. $\%$ primer accionista & 0.662 & 0.355 & -0.019 & 0.011 & 1.000 & & & & \\
\hline 4. EBITDA 2006-2008 & 0.329 & 1.283 & $-0.164 * *$ & -0.025 & 0.007 & 1.000 & & & \\
\hline 5. Propiedad Familiar & 0.072 & 0.259 & -0.067 & $-0.022 *$ & $0.024 *$ & -0.010 & 1.000 & & \\
\hline 5. Crecimiento empleado & s0.334 & 0.784 & $-0.104 * *$ & $0.077 * *$ & -0.018 & 0.002 & -0.0121 .000 & & \\
\hline 7. Crecimiento activos & 0.536 & 0.537 & $-0.136 * *$ & 0.003 & 0.030 & 0.005 & $-0.017-0.003$ & 1.000 & \\
\hline 3. Crecimiento ventas & 0.488 & 0.970 & $-0.098 * *$ & -0.026 & 0.012 & -0.005 & -0.0110 .034 & $0.075 * *$ & 1.000 \\
\hline
\end{tabular}

\section{Figura 1}

Efecto interacción del tamaño y edad de la empresa.
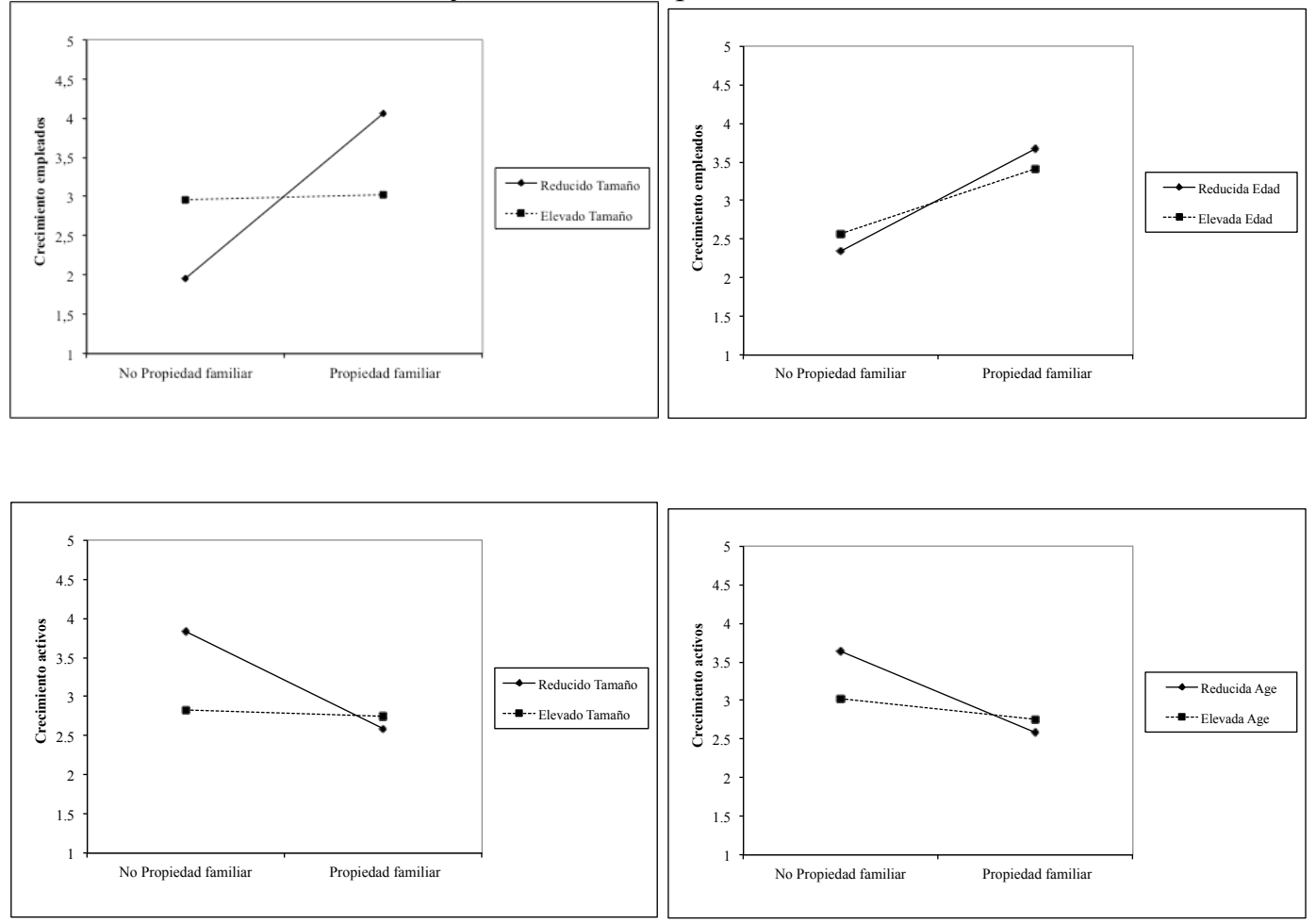
sobre el crecimiento del número de empleados $(b=0.545 ; p<0.001)$. Sin embargo, las interacciones entre la propiedad familiar y el tamaño y la edad de las empresas son significativas, ambos con un coeficiente negativo (respecto al tamaño: $\mathrm{b}=-0.510 ; \mathrm{p}<$ 0.001 ; respecto a la edad: $b=-0.123 ; p<0.001)$. Con el fin de comprender mejor estos resultados hemos procedido a representarlos gráficamente en la figura 1. Como puede verse, las empresas familiares de menor tamaño son responsables de las mayores tasas de crecimiento en el numero de trabajadores a pesar de los resultados negativos en años precedentes. Por el contrario, en el caso de las empresas más grandes la estructura de propiedad no parece ejercer apenas influencia sobre el crecimiento del número de trabajadores de la empresa. Respecto a la edad de la empresa, la figura 1 pone de manifiesto que las empresas familiares, especialmente las más maduras, muestran tasas de crecimiento en el número de empleados superiores a las no familiares.

La segunda variable dependiente es la evolución de la partida de activos de la empresa. En este caso, de nuevo el tamaño y la edad muestran una influencia negativa y significativa (para el tamaño: $b=-0.213 ; p<0.001$; para la edad: $b=-0.113 ; p<0.001)$. Como en el caso del empleo, las empresas más pequeñas $\mathrm{y}$ jóvenes incrementan o mantienen sus niveles de activos, mientras que las más grandes y antiguas muestran una tendencia a reducirlos. Sin embargo, el hecho de que una familia controle la propiedad de la empresa ejerce un impacto negativo sobre la evolución de los activos $(b=-$ $0.331 ; \mathrm{p}<0.001)$. Las empresas familiares muestran, por tanto, una mayor propensión a

\section{Tabla 2}

Análisis de regresión.

\begin{tabular}{|c|c|c|c|c|c|c|}
\hline \multirow[b]{2}{*}{ Constante) } & \multicolumn{3}{|c|}{ Crecimiento empleados } & \multicolumn{3}{|c|}{ Crecimiento activos } \\
\hline & $0.870 * * *$ & $0.786 * * *$ & 0.034 & $3.419 * * *$ & $4.181 * * *$ & $4.948 * * *$ \\
\hline Fabricación & 0.043 & 0.040 & 0.036 & 0.032 & 0.027 & 0.022 \\
\hline Josntrucción & 0.001 & 0.001 & 0.001 & 0.054 & 0.045 & 0.037 \\
\hline Jomercio & 0.086 & 0.081 & 0.071 & 0.091 & 0.076 & 0.063 \\
\hline Jervicios & 0.036 & 0.034 & 0.030 & 0.106 & 0.088 & 0.073 \\
\hline -nTamaño & $-0.108 * * *$ & $-0.102 *$ & $-0.004 *$ & $-0.141 * * *$ & $-0.156 * * *$ & $-0.213 * * *$ \\
\hline nEdad & $-0.048 * *$ & $-0.042 *$ & $-0.010 *$ & $-0.054 * *$ & $-0.131 * *$ & $-0.113 * *$ \\
\hline $\mathrm{n} \mathrm{N}^{\mathrm{o}}$ Accionistas & -0.016 & -0.018 & -0.003 & 0.016 & 0.023 & 0.050 \\
\hline 1\%first shareholder & -0.030 & -0.030 & 0.001 & 0.033 & 0.033 & $0.080 *$ \\
\hline IBITDA 2006-2008 & -0.010 & -0.013 & -0.002 & -0.017 & -0.009 & -0.012 \\
\hline Jropiedad Familiar & & 0.031 & $0.545^{* * *}$ & & $-0.075 * *$ & $-0.331 * *$ \\
\hline Tamiliar x Tamaño & & & $-0.510 * * *$ & & & $0.290 * *$ \\
\hline amiliar x Edad & & & $-0.123 * *$ & & & $0.201 *$ \\
\hline Familiar x No Accionistas & & & -0.003 & & & -0.060 \\
\hline Familiar $\mathrm{x}$ Primer accionist & & & -0.048 & & & $-0.065 \dagger$ \\
\hline amiliar x EBITDA & & & -0.031 & & & -0.007 \\
\hline $22 \mathrm{adj}$. & 0.010 & 0.010 & 0.022 & 0.018 & 0.023 & 0.028 \\
\hline ;ig. & 0.000 & 0.001 & 0.000 & 0.000 & 0.000 & 0.000 \\
\hline nc R2 & $0.013 * * *$ & 0.001 & $0.014 * * *$ & $0.021 * * *$ & $0.005 * *$ & $0.008 *$ \\
\hline
\end{tabular}




\section{Figura 2}

Efecto moderador de la estructura de propiedad.

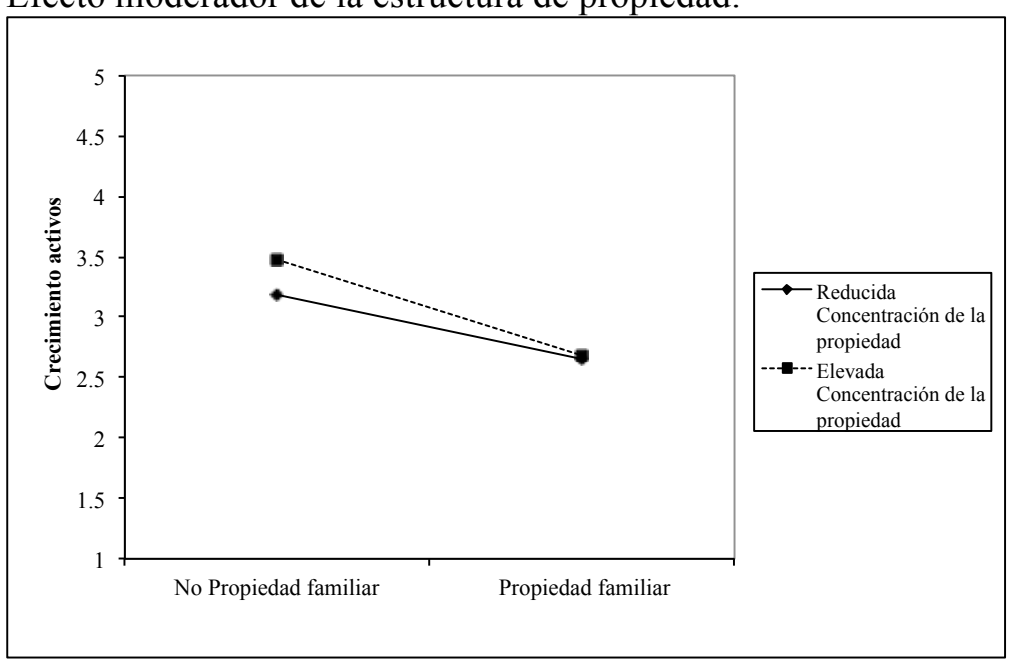

reducir sus activos a través de desinversiones. Asimismo, las interacciones entre el carácter familiar de la empresa por un lado, y del tamaño y edad de la misma por otro, son igualmente significativos (para el tamaño: $b=0.290 ; p<$ 0.001 ; para la edad: $b=0.201 ; p<0.001)$, en este caso, con un coeficiente de regresión positivo. Igualmente, la concentración de la propiedad ofrece un efecto positivo sobre la evolución de los activos $(b=0.080 ; p<0.01)$, y su efecto en interacción con la naturaleza familiar de la empresa es negativo $y$ significativo $(b=-0.065 ; p<0.05)$. Como en el caso anterior, con el fin de interpretar mejor estos resultados, hemos representado gráficamente los efectos moderadores significativos también en la figura 1.

Respecto al tamaño, la figura pone de manifiesto que las empresas pequeñas $\mathrm{y}$ familiares son las que presentan unos mayores niveles de desinversión. Las empresas no familiares y las familiares pero de mayor tamaño ofrecen una evolución más estable en sus niveles de activos, a pesar de los malos resultados precedentes. Algo similar encontramos en relación a la antigüedad de la empresa. La figura 1 permite comprobar cómo las empresas familiares más jóvenes tienden a reducir sus activos más que las más maduras así como que las empresas no familiares. Finalmente, en la figura 2 hemos representado el efecto interacción entre la naturaleza familiar de la empresa y el grado de concentración de la propiedad. En este caso, podemos observar que las empresas familiares con una elevada concentración en la propiedad (propio de empresas familiares en sus primeros niveles de su ciclo de vida) son las más proclives a reducir sus niveles de activos ante unos resultados negativos, frente a las empresas familiares con una mayor dispersión en su estructura de propiedad (propio de las empresas familiares en niveles más avanzados).

\section{Discusión y Conclusiones}

El presente trabajo analiza como la estructura de propiedad influencia el modo en que las empresas afrontan estrategias de reestructuración ante resultados negativos a lo largo de varios años. En concreto, se investigan las diferencias entre las empresas familiares y no familiares en el modo en que reaccionan ante una serie de resultados negativos. En esta investigación defendemos que el carácter familiar de la empresa puede ejercer tanto una influencia positiva como negativa en relación con la intensidad y el sentido de los procesos de reestructuración ante resultados negativos. En primer lugar, la naturaleza familiar puede favorecer el desarrollo rápido de estrategias de reajuste o cambio de rumbo, en la medida en que estas empresas han demostrado una mayor orientación emprendedora (Melin y Nordqvist, 2010; Casillas, Moreno y Barbero, 2010). Esta orientación emprendedora no sólo permite a este tipo de empresa identificar y explotar nuevas oportunidades de negocio, sino también les proporciona una mayor capacidad de reacción ante resultados poco satisfactorios a través de 
procesos de desinversión o de reasignación de recursos. Sin embargo, los propietarios familiares suelen mostrar un mayor compromiso con la empresa familiar como pone de manifiesto el enfoque basado del stewarship (Eddleston y Kellermanns, 2007; Miller et al., 2008; Eddleston et al., 2012). Este enfoque pone de manifiesto que las empresas familiares presentan una mayor orientación al stewarship que se ve reflejado en una mayor orientación hacia el largo plazo, hacia sus empleados y hacia sus clientes (Miller et al., 2008). Esta orientación se puede poner de manifiesto en una tendencia a evitar la toma de decisiones drásticas que puedan afectar a la organización, trato de mantener los empleos, productos y estrategias tradicionales de la empresa. En este trabajo se proponen asimismo, una serie de variables moderadoras, como el tamaño, la edad y en el nivel de concentración en la estructura de propiedad.

Los resultados obtenidos muestran que la propiedad familiar influye sobre el modo en que las empresas reaccionan ante los malos resultados. Así, se observan diferencias entre dicha reacción por parte de empresas familiares y no familiares, de forma que las primeras tienden a mantener o aumentar puestos de trabajo, mientras que reducen sus activos a través de procesos de desinversión. Asimismo, hemos comprobado que esta relación se da con mayor intensidad en el caso de las empresas familiares de menor tamaño y edad. De este modo, podemos contrastar parcialmente la primera de las hipótesis propuestas. En concreto, nuestros resultados contrastan la hipótesis 1a (ante resultados negativos, las empresas familiares tienden a realizar procesos de ajuste mas intensos que las empresas no familiares), en relación con los activos. Sin embargo, en relación con el número de trabajadores, los resultados contrastan la hipótesis alternativa, esto es, la hipótesis $1 \mathrm{~b}$ (Ante resultados negativos, las empresas familiares tienden a realizar procesos de ajuste menos intensos que las empresas no familiares).

Respecto a la hipótesis 2, (la relación entre estructura de propiedad y las estrategias de reestructuración será más intensa de las empresas de mayor tamaño y edad), nuestros resultados rechazan dicha hipótesis, encontramos una relación opuesta a la inicialmente propuesta. Este resultado puede ser explicado por la mayor flexibilidad de las empresas más pequeñas y jóvenes frente al peso de la inercia de las empresas más grandes y maduras, que les dificulta poner en práctica estrategias reactivas ante resultados negativos. La mayor agilidad de las empresas más pequeñas y jóvenes les permite adoptar medidas más rápidas y radicales para atajar la decadencia en los resultados, frente a las empresas más maduras y de mayor tamaño que pueden encontrar una mayor resistencia a este tipo de medidas.

Finalmente, en relación con la hipótesis 3 (la relación entre estructura de propiedad $y$ estrategias de reestructuración será más intensa cuando el nivel de concentración de la propiedad es más elevado que cuando la propiedad está muy dispersa), hemos encontrado que el nivel de concentración de la propiedad únicamente modera en el caso de la evolución en el nivel de activos. Por esta razón, podemos afirmar que dicha hipótesis 3 ha sido parcialmente confirmada.

El presente trabajo identifica aspectos interesantes en relación con el modo en que las empresas afrontan periodos de resultados decrecientes, tan habitual en momentos como el actual, donde el dinamismo y la hostilidad del entorno alcanzan cotas muy elevadas. No obstante, la investigación adolece de ciertas limitaciones. En primer lugar, desde una perspectiva teórica, se hace necesario profundizar en las aportaciones de las visiones basadas en el stewarship (Miller et al., 2008). En segundo lugar, hemos medido las estrategias de reestructuración a través de la evolución de dos indicadores a lo largo de un año. Aunque el periodo de resultados negativos ha sido medido en un periodo de tres años, anteriores al del efecto de la reestructuración sería deseable contar con un lapso temporal más dilatado para comprobar la robustez de los resultados obtenidos. En tercer lugar, hemos empleado una muestra de un solo país, sin que seamos capaces de poder comprobar en qué medida afecta el 
contexto nacional y si es posible generalizar los resultados a otros países. Y finalmente, hemos empleado fundamentalmente medidas financieras, obtenidas a través de fuentes secundarias de información, siendo deseable poder contar con información más detallada e interna de los procesos de toma de decisiones relativas a la reestructuración.

No obstante, hemos identificado un ámbito de estudio que requiere de más investigación, especialmente en el contexto de dificultades económicas en el que se desenvuelven las empresas actualmente. Nuestros resultados, al no ser del todo concluyentes, abren la vía a nuevas investigaciones que permitan comprender mejor como afecta la estructura de propiedad en los procesos de toma de decisiones, así como diferentes dimensiones del carácter familiar de una empresa, más allá de su control de la propiedad. En este sentido, sería interesante desarrollar investigaciones de corte longitudinal, que permitan comprender los aspectos intrínsecamente dinámicos de estos procesos. Asimismo, sería conveniente incorporar el análisis de dimensiones organizativas (como la flexibilidad, las estructuras de gobierno, las características de la alta dirección, etc.) y dimensiones en el ámbito familiar (implicación de la familia en el negocio, nivel generacional, orientación emprendedora de la familia, etc.) como variables explicativas, moderadoras o mediadoras que permitan comprender mejor las diferencias en la reacción ante resultados negativos de las empresas. Finalmente, el desarrollo de análisis de la supervivencia de las empresas ante estos resultados podría aportar luz acerca de la eficacia de estos procesos de reestructuración.

La presente investigación sólo ofrece un primer paso en una línea potencialmente interesante para futuros desarrollos, teóricos y empíricos. Hemos encontrado diferencias en los modos de reestructuración entre empresas familiares y no familiares. Confiamos que en el futuro, nuevos trabajos aporten nuevo conocimiento sobre este fenómeno al tiempo que oriente a los directivos sobre cómo mejorar sus capacidades para gestionar empresas con un declive en sus resultados económicos y financieros.

\section{Bibliografía}

Abor, J., Graham, M. and Yawson, A. (2011). Corporate Governance and restructuring activities following completed bids. Corporate Governance, An International Review, 19(1), 61-76.

Aguilera, R. and Crespí, R. (2012). Firm family firms: Current debates of corporate governance in family firms. Journal of Family Business Strategy, 3, 66-69.

Alchian, A. and Demsetz, H. (1972). Production, information costs, economic organization. American Economic Review, 62(5), 777-795.

Ansoff, H.I. (1957). Strategies for Diversification. Harvard Business Review, 35(5), 113-124.

Bammens, Y., Voordeckers, W. and Van Gils, A. (2011). Boards of Directors in Family Businesses: A literature Review and Research Agenda. International Journal of Management Reviews, 13, 134-152.

Barker, V.L. and Mone, M.A. (1994). Retrenchment: Cause of turnaround or consequence of decline? Strategic Management Journal, 15(5), 395-405.

Barney, J. B. (1991). Firm Resources Sustained Competitive Advantage. Journal of Management, 17(1), 99-120.

Behn, R.D. (1980). Leadership for cut-back management: The use of corporate strategy. Public Administration Review, 40(6), 613.

Boyle, R.D. Desai, H.B. (1991). Turnaround strategies for small firms. Journal of Small Business Management, 29(3), 33-42.

Casillas, J.C., Moreno, A.M. and Barbero, J.L. (2010). A Configurational Approach of the Relationship Between Entrepreneurial Orientation Growth of Family Firms. Family Business Review, 23(1), 27-44.

Cater III, J.J. and Schwab, A. (2008). Turnaround strategies in established small family firms. Family Business Review, 21(1), 31-50.

Chrisman, J.J., Chua, J.H. and Steier, L.P. (2003). An introduction to theories of family business. Journal of Business Venturing, 18(4), 441448. 
Chua, J.H., Chrisman, J.J. and Steier, L.P. (2003). Extending the theoretical horizons of family business research. Entrepreneurship Theory Practice, 27(4), 331-338.

Collis, D.J. and Montgomery, C.A. (1998). Crating corporate advantage. Harvard Business Review. 76(3), 70-84.

D'Aveni, R.A. (1989). The aftermath of organizational decline: A longitudinal study. Academy of Management Journal, 32(3), 577-605.

Di Primio, A. (1988). When turnaround management works. The Journal of Business Strategy, 9(1), 61-64.

Gedajlovic, E., Lubatkin, M.H. Schulze, W.S. (2004). Crossing the threshold from founder management to professional management: a generational perspective. Journal of Management Studies, 41(5), 899-912.

Goldeng, E., Grünfeld, L.A. and Benito, G.R.G. (2008). The performance differential between private state owned enterprises: The roles of ownership, management market structure. Journal of Management Studies, 45(7), 1244-1273.

Hall, W.K. (1980). Survival strategies in a hostile environment. Harvard Business Review, 58(5), 75 85 .

Harrison, J.S., Hitt, M.A., Hoskisson, R.E. and Irel, R.D. (2001). Resoruces complementary in business combinations: Extending the logic to business combinations. Journal of Management, 27(6), 679-690.

Haynes, M., Thompson, S. and Wright, M. (2003). The determinants of corporate divestment: Evidence from a panel of UK firms. Journal of Economic Behavior \& Organization, 52, 147-166.

Hofer, C. W. and Schendel, D. (1978). Strategy Formulation: Analytical Concepts. St. Paul, MN: West Publishing.

Ibrahim, A.B., Soufani, K. and Lam, J. (2001). A study of succession in a family firm. Family Business Review, 14(3), 245-258.

International Monetary Found. (2010) Oslo Conference.

Jensen, M. C. (1993). The modern industrial revolution, exit, the failure of internal control systems. The Journal of Finance, 48(3), 831-880.

Jensen, M.C. and Meckling, W.H. (1976). Theory of the firm: managerial behavior, agency costs ownership structure. Journal of Financial Economics, 3(4), 305-360.

Jensen, M.C. (1986). Agency costs of free cash flow, corporate finance, takeovers. The American Economic Review, 76(2), 323-329.

Kierulff, H. and Petersen, H.L. (2009). Finance is everything: Advice from turnaround managers. Journal of Business Strategy, 30(6), 44-51.

Knott, A.M. and Posen, H.E. (2005). Is failure good? Strategic Management Journal, 26(7), 617641.

Kotler, P. (2000). Marketing Management, the millenium edition, international edition. Upper Saddle River, New Jersey: Prentice Hall.

Lieberman, M.B. (1987). Strategies for Capacity Expansion. Sloan Management Review, 28(4), 1928.

Miller, D., Breton-Miller, I. and Scholnick, B. (2008). Stewardship vs. stagnation: An empirical comparison of small family and non-family business. Journal of Management, 45(1), 51-78.

Mitchell, J.R., Hart, T.A., Valcea, S. and Townsend, D.M. (2009). Becoming the boss: Discretion postsuccession success in family firms. Entrepreneurship, Theory Practice, 33(6), 12011218.

Moreno, A.M. and Casillas, J.C. (2008). Entrepreneurial Orientation Growth of SMEs: A causal model. Entrepreneurship Theory Practice. 32(3), 507-528.

Morrow Jr, J.L., Johnson, R. A. and Busenitz, L.W. (2004). The effects of cost asset retrenchment on firm performance: The overlooked role of a firm's competitive enrivonment. Journal of Management, 30(2), 189-208.

Murphy, J. (1986). First aid for unhealthy companies. Australian Accountant, 56(4), 29.

Nordqvist, M. and Melin, L. (2010). Entrepreneurial families family firms. Entrepreneurship. Regional Development, 22(3-4), 211-239.

Nordqvist, M., Habbershon, T.G. and Melin L. (2008). Transgenerational entrepreneurship: Exploring entrepreneurial orientation in family firms. In H. Landstrom, D. Smallbone, H. Crijns and E. Laveren (Ed.), Entrepreneurship, sustainable growth and performance: Frontiers in European entrepreneurship research. London: Edward Elgar. 
Pearce II, J. A. and Robbins, D.K. (2008). Strategic transformation as the essential last step in the process of business turnaround. Business Horizons, 51(2), 121-130.

Pearce II, J.A. and Robbins, D.K. (1994a). Entrepreneurial recovery strategies of small market share manufacturers. Journal of Business Venturing, 9(2), 91-108.

Pearce II, J.A. and Robbins, D.K. (1994b). Retrenchment remains the foundation of business turnaround. Strategic Management Journal, 15(5), 407-417.

Pearce II, J.A. and Robbins, K. (1993). Toward improved theory research on business turnaround. Journal of Management, 19(3), 613-636.

Penrose, E. T. (1959). The theory of the growth of the firm. New York: John Wiley.

Porter, M.E. (1980). Competitive Strategy. New York: Free Press.

Porter, M.E. and Spence, A.M. (1982). The capacity expansion process in a growing oligopoly: The case of Corn Wet Milling. In J.J. McCall (Ed.), Economics of Information Uncertainty. Chicago: University of Chicago Press.

Rahul, K. Hitt, M.A. (1998). Research notes communications linking corporate strategy to capital structure: Diversification strategy, type source of financing. Strategic Management Journal, 19(6), 601-610.

Rahul, K. (1996). Explaining firm capital structure: The role of agency theory vs. transaction cost economics. Strategic Management Journal, 17(9), 713-728.

Robbins, D.K. and Pearce II, J.A. (1992). Turnaround: Retrenchment Recovery. Strategic Management Journal, 13(5), 287-309.

Robbins, D.K. and Pearce II, J.A. (1993). Entrepreneurial retrenchment among small manufacturing firms. Journal of Business Venturing, 8(4), 301-318.

Rumelt, R.P. (1974). Strategy, structure, economic performance. Cambridge, Massachussets: Harvard Business School Press.

Schulze, W. G., Lubatkin, M. H. and Dino, R. N. (2003). Exploring the agency consequences of ownership dispersion among the directors of private family firms. Academy of Management Journal, 46(2), 179-194.

Shanker, M.C. and Astrachan, J.H. (1996). Myths realities: Family businesses contribution to U.S. economy- A framework for assessing family business statistics. Family Business Review, 9(4), 107-119.

Sharma, P. (2003). Stakeholder mapping technique: Toward the development of a family firm typology. Laurie Business \& Economics, Waterloo: Canada. (Ac-Acta Wasaensia 145 cessed on August 29,

https://webaccess.wlu.ca/documents/842/2003-01MOB.pdf.

Sharma, P. (2004). An overview of the field of family business studies: Current status directions for the future. Family Business Review, 17(1), 1-36.

Ward, J.L. (1987). Keeping the family business healthy: How to plan for continuing growth, profitability, family leadership. San Francisco, CA: Jossey-Bass.

Zahra, S. A., Hayton, J. C. Salvato, C. (2004). Entrepreneurship in family vs. non-family firms: A resource-based analysis of the effect of organizational culture. Entrepreneurship Theory Practice, 28(4), 363-381. 